\title{
Emergency management plan for paediatric patients with tracheostomies during the COVID-19 pandemic
}

\author{
Beckie Petulla ${ }^{1}$, Emma Ho ${ }^{1}$, Emma Sov ${ }^{1}$, and Marlene Soma ${ }^{1}$ \\ ${ }^{1}$ Sydney Children's Hospital Randwick
}

November 8, 2021

\begin{abstract}
Objectives: Paediatric patients living with tracheostomies are a medically vulnerable group. During the COVID-19 pandemic, there may be apprehension about their susceptibility to SARS-CoV-2 infection with unknown consequences. Healthcare workers managing this cohort can be anxious about viral transmission from respiratory secretions and aerosols emerging from the open airway. Our objective is to share a systematic approach to minimise incidental mismanagement, avoid iatrogenic airway injury, reduce aerosolisation and decrease staff exposure when treating these patients. Methods: A COVID-19 emergency management plan was created for paediatric patients with tracheostomies in the event of presentation with unknown, suspected or proven SARS-CoV-2 infection. Three documents were developed: a generic tracheostomy management plan detailing troubleshooting measures to reduce air leak from around the tube; a personalised management plan with customised recommendations; a guide for tracheostomy tube change with emphasis on minimising aerosol production. Results: Our plan was distributed to 31 patients (age range 11 months - 17 years) including 23 (74.2\%) with uncuffed tubes and 9 (29\%) on long term ventilation. There have been 10 occasions in which the plan was utilised and influenced care, including 4 situations where successful troubleshooting avoided tube manipulation and 6 situations where an uncuffed tube was safely replaced with a pre-selected cuffed tube to reduce air leak. Conclusions: A structured approach to emergency presentations during the COVID-19 pandemic may safeguard paediatric patients from unnecessary manipulation of their tracheostomy tube and airway trauma, as well as provide guidance to minimise viral exposure and allow provision of expeditious care.
\end{abstract}

\section{Emergency management plan for paediatric patients with tracheostomies during the COVID- 19 pandemic}

Beckie Petulla ${ }^{1}$ RN, Emma Ho ${ }^{2}$ MD, Emma Sov ${ }^{1}$ BAppSc (OT), Marlene Soma ${ }^{2,3}$ MBBS FRACS

${ }^{1}$ Sydney Children's Hospital, Randwick, Australia

2 Department of Otolaryngology, Sydney Children's Hospital, Randwick, Australia

${ }^{3}$ School of Women and Children's Health, University of New South Wales Sydney

No financial support or grants were received for the conduct of this study

Principal author:

Beckie Petulla

Complex and Artificial Airways Clinical Nurse Consultant

Sydney Children's Hospital

High St

Randwick, NSW 2031 
Australia

Phone: +61 (2) 9382-1111

Fax: $+61(2)$ 9382-0725

Email: beckie.petulla@health.nsw.gov.au

Keywords: COVID-19, paediatric tracheostomy, risk management, quality improvement, aerosol generating procedure

Abbreviated title: COVID-19 plan for paediatric tracheostomy patients

\section{ABSTRACT}

Objectives: Paediatric patients living with tracheostomies are a medically vulnerable group. During the COVID-19 pandemic, there may be apprehension about their susceptibility to SARS-CoV-2 infection with unknown consequences. Healthcare workers managing this cohort can be anxious about viral transmission from respiratory secretions and aerosols emerging from the open airway. Our objective is to share a systematic approach to minimise incidental mismanagement, avoid iatrogenic airway injury, reduce aerosolisation and decrease staff exposure when treating these patients.

Methods: A COVID-19 emergency management plan was created for paediatric patients with tracheostomies in the event of presentation with unknown, suspected or proven SARS-CoV-2 infection. Three documents were developed: a generic tracheostomy management plan detailing troubleshooting measures to reduce air leak from around the tube; a personalised management plan with customised recommendations; a guide for tracheostomy tube change with emphasis on minimising aerosol production.

Results: Our plan was distributed to 31 patients (age range 11 months - 17 years) including 23 (74.2\%) with uncuffed tubes and $9(29 \%)$ on long term ventilation. There have been 10 occasions in which the plan was utilised and influenced care, including 4 situations where successful troubleshooting avoided tube manipulation and 6 situations where an uncuffed tube was safely replaced with a pre-selected cuffed tube to reduce air leak.

Conclusions: A structured approach to emergency presentations during the COVID-19 pandemic may safeguard paediatric patients from unnecessary manipulation of their tracheostomy tube and airway trauma, as well as provide guidance to minimise viral exposure and allow provision of expeditious care.

\section{INTRODUCTION}

SARS-CoV-2 is a virus that causes a life-threatening disease known as COVID-19. The World Health Organisation declared COVID-19 a global pandemic on March 11, 2020 . Transmission is facilitated primarily via respiratory droplets ${ }^{2}$. Infection can be spread by asymptomatic, pre-symptomatic and symptomatic carriers of the virus. Otolaryngology groups are recommending that a higher level of personal protective equipment (PPE) should be used when performing aerosol-generating procedures (AGP) ${ }^{3-6}$. This is due to the increased risk of infectious transmission secondary to high oral and nasal viral titres and the unknown potential for aerosol generation during procedures ${ }^{4}$. Tracheostomy and endotracheal suctioning can especially result in the spread of small aerosols containing viable pathogens ${ }^{6}$.

Children living with new or longstanding tracheostomies are a medically vulnerable group. This cohort of patients often have complex co-morbidities in addition to their need for an artificial airway. Care providers in hospitals and in the community are not always comfortable with managing these patients when they have acute presentations. In the current pandemic, there can be considerable apprehension from parents and caregivers about their child being more susceptible to the virus, fear about consequences of infection and concern about navigating the healthcare system during periods of restricted access ${ }^{7,8}$.

Healthcare workers are potentially at an increased risk of viral exposure and transmission when managing a patient with an open airway. Limiting the spread of all droplet-borne pathogens, not just SARS-CoV-2, 
is important as the development of any respiratory tract symptoms can impact upon the medical workforce and have implications for close contacts of the patient and staff in these times. AGPs often occur after hours or in emergency situations, moreover they may also occur at peripheral hospitals where access to specialist staff and support is limited. A clear team checklist and simple instructions may guide healthcare workers regarding when to perform AGP's in situations when COVID-19 is unknown, suspected or confirmed, as well as prepare them to perform AGPs safely. With written clarification, the proceduralists should feel confident and empowered to undertake AGP in a controlled manner, reducing the risks to the patient and the health care team.

This project was initiated when a paediatric patient had an incorrectly sized cuffed tube inserted in an Emergency Department (ED) in response to concerns about excessive aerosol generation from the tracheostomy tube and stoma whilst awaiting results for SARS-CoV-2 serology. Since that event, a simple COVID-19 emergency instruction plan was created for care providers outlining management principles for this group of patients during the pandemic to improve quality of care. It has been developed by the Clinical Nurse Consultant for Paediatric Complex and Artificial Airways in close collaboration with the Otolaryngology, Children's Intensive Care Unit and the Paediatric Emergency Departments.

Our emergency management plan for these patients has several benefits. Firstly, a clear algorithm to troubleshoot around the existing tube to minimise air leak if SARS-CoV-2 infection is unknown, suspected or confirmed. Secondly, if the initial strategies are not sufficient, then a considered approach to decrease the risk of patient morbidity from iatrogenic injury by identifying an appropriate alternative tracheostomy tube for the individual. Thirdly, a clear and structured plan is provided to minimise AGP risk to health care workers whilst undertaking manipulation of the tracheostomy tube in these patients if needed, to protect them from potential infectious exposure and allow the provision of expeditious care.

\section{Materials and Methods}

The authors work at a large tertiary referral children's hospital located on a shared campus with a tertiary women's hospital that includes a neonatal intensive care unit, tertiary adult's hospital and a private hospital that also has a special care unit for neonates. Ethics approval was not required for this project as it was a quality improvement proposal adapted from an existing hospital policy developed by the Children's Intensive Care Unit ${ }^{9}$ with permission granted to modify it.

A 3-document resource was developed for care providers managing paediatric patients with tracheostomies during the COVID-19 pandemic. Each document was only 1 page long and printed in colour to make the key points identifiable and straightforward to read. It was written in plain language to be easily understood by families, nursing, allied health and medical staff, irrespective of their background training in paediatric tracheostomy care provision.

The first document titled "Tracheostomy Management Plan during COVID-19 Pandemic" is a generic overview applicable to all patients in this group (Figure 1). When presenting for acute care, a COVID-19 viral swab and full respiratory panel should be collected as a matter of urgency for processing. Establishing positivity for COVID-19 in this cohort has implications for where the child should be managed and the appropriate levels of PPE healthcare workers require. Respiratory secretions from the tracheostomy are adequate for sampling and can avoid distressing the child from nasopharyngeal or oropharyngeal swab collection.

If the patient has a cuffed tracheostomy tube, the cuff should be inflated to minimise any leak and the cuff pressure checked. If there are concerns that the existing cuff is not functioning properly, consideration could be given to replacement with a cuffed tube in working order. Patients who use an uncuffed tube should also be assessed for a significant leak around the stoma. In this is identified, it may be appropriate to temporarily change to a pre-selected cuffed tube for the individual in a careful and controlled manner. Once the leak has been reduced, two split surgical dressings are recommended to be placed around the stoma and a surgical mask is placed over the mouth and nose of the patient if it can be tolerated. The use of appropriate-sized viral filters based on weight and tidal volumes is advised for all tracheostomy patients during these presentations. 
The second document titled "Patient's Personalised Management Plan during COVID-19 Pandemic" was designed to be customisable for the individual and includes information about the current tracheostomy tube in use and any specific recommendations for change (Figure 2). This was necessary as paediatric patients may have a particular size or type of tube in situ chosen for specific reasons (e.g. unique anatomy, need for airway protection, require long term ventilation, on decannulation pathway etc.). If there are concerns about excessive aerosol or secretion generation from the stoma around an uncuffed tracheostomy tube it may be necessary in some circumstances to temporarily change to a cuffed tracheostomy tube. This is often not a straightforward swap from an uncuffed to an equivalent sized cuffed tube as there are fewer cuffed tubes in paediatric sizes on the market and they can be composed of different materials, with different lengths, outer diameters and angulation. Not all care facilities are expected to be stocked with such a range of tubes.

To generate the personalised plan, all paediatric patients with a tracheostomy currently being managed by our service were identified and reviewed. Demographics and details about their indication for tracheostomy, current tube size and type, status of the stoma, specific anatomical concerns and need for long term ventilation were collated. The primary Otolaryngologist was consulted for each patient to confirm individualised recommendations to avoid traumatising the stoma or the internal airway in the event that temporary change to an alternative tube was required. Each patient was given this pre-selected tracheostomy tube to keep in their emergency kit for such a situation.

The third document titled "Changing a tracheostomy tube for suspected or proven COVID-19 infection" entails the steps recommended to undertake this procedure if required (Figure 3). The guide is intended to provide a systematic approach for care providers once the decision to change the tracheostomy tube has been agreed upon by the treating specialists from our hospital. It covers team preparation, equipment preparation, patient preparation and the technique itself with an emphasis on risk mitigation for both the patient and the staff involved ${ }^{10,11,12}$.

The procedure should preferably occur in a negative pressure single room with full airborne precautions and PPE. Prior to commencing, the team should be assembled, roles and steps of the intervention defined, positioning of the patient and team members discussed, all equipment checked, drugs available (if needed) and arranged in a way to ensure the procedure is performed efficiently and escalation of emergency management can be initiated without the need to exit the negative pressure room. This includes reviewing access to suction, oxygen, monitoring equipment and waste disposal. Pre-medication may be considered to reduce patient anxiety, coughing and excessive movement. The parent/caregiver should be involved as they are likely the most skilled operator in paediatric tracheostomy changes for the child. The second assistant aids in securing neck ties and positioning the new tube whereas the third assistant manages sedation, additional and emergency equipment as required. Having another person outside the room who can be easily contacted (e.g. via writing on a whiteboard or speaking on a telephone or walkie talkie checked prior to commencement) can be beneficial as once in PPE in a confined space, it can be challenging to communicate with others outside if more help or items are needed.

The patient should have appropriate cardiorespiratory monitoring established including end tidal $\mathrm{CO}_{2}$ if available during manipulation of the tracheostomy site. Inline suctioning should be prepared and ready to utilise for the tracheostomy tube and oral suction catheters accessible if required. The patient should be preoxygenated for 5 minutes prior and positioned to expose the tracheostomy. The tube change is undertaken and once it has occurred, post procedure airborne precautions are maintained for 15-30 minutes as per the local hospital policies.

The family of each of our patients were contacted and provided education about the COVID-19 emergency management plan, and their established personalised emergency tracheostomy kits were updated to include a hard copy of this new resource and appropriate supplies if required. In addition, the caregivers were provided with an electronic copy and the personalised management plan for each patient was uploaded to the hospital electronic medical records.

No direct financial support or grants were received for the conduct of this study. For those patients in 
whom it was recommended temporarily changing to a different type or size of tracheostomy tube to reduce aerosol production, the tubes were supplied to the individuals by Enable NSW (a government department that provides assistive technology and related services to people with specific, short term or ongoing health needs to assist them to live safely at home).

\section{Results}

At present, our service manages 31 paediatric patients (age range 11 months to 17 years) with tracheostomies in a variety of different care settings, though the majority (93.5\%) are cared for at home. $23(74.2 \%)$ patients have an uncuffed tracheostomy tube in situ and $9(29 \%)$ patients require long term ventilation. Since the introduction of this initiative, there have been 10 presentations in which our COVID-19 emergency management plan has been actively utilised and influenced care. On 4 occasions, the troubleshooting recommendations were followed successfully without the need to escalate to a tube change. 6 tube changes were undertaken with safe transition from an uncuffed to the individual's pre-selected cuffed tube to minimise air leak. This included one patient who has been on long term ventilation who required intervention on 3 separate occasions. The cuffed tube remained in situ for these patients for an average of 4.3 days (range 0.5 to 14 days) depending on the clinical condition of the child. There have been no incidents of stomal or airway injury during the insertion or removal of the temporary cuffed tube. Fortunately none of our paediatric tracheostomy patients have been positive for SARS-Co-V2 to date. To the best of our knowledge, no patients have required tube changes outside of our protocol recommendations in their personalised plan.

Since the introduction of our management plan, the Otolaryngology team and Clinical Nurse Consultant have been contacted on multiple occasions when our paediatric patients with tracheostomies have presented to EDs around the state. Direct advice has been given, risk reduction measures have been recommended and instituted early. Families have reported feeling more comfortable about their child's care whilst awaiting clearance for COVID-19. Feedback from healthcare professionals working in EDs to date has been overwhelmingly positive. An ED specialist at a peripheral hospital reported that one of our patients with the COVID-19 emergency plan was managed primarily by the parents as they had been well educated in this regard. Healthcare worker exposure was minimised and support from the tertiary ENT team was accessed quickly, reducing local staff anxiety, improving patient outcomes and patient flow. In this case, troubleshooting was effectively initiated based on the suggestions provided in our management plan to reduce the air leak around the tracheostomy tube while awaiting rapid COVID-19 testing results such that the need for conversion to a cuffed tracheostomy cuffed tube was avoided.

\section{DISCUSSION}

Children who require a tracheostomy as part of their management are a complex set of patients with increased medical needs. Respiratory tract illnesses in this group can have variable manifestations depending on the pathogen involved, the child's immune status, underlying pulmonary disease and ability to effectively manage secretions. The impact of COVID-19 on these patients is unknown with only one reported case in the literature of hospitalisation ${ }^{13}$ and two children with milder illness ${ }^{14}$. Fortunately the worldwide experience is of a generally less severe illness and lower mortality rate in children than in adults overall. There are concerns however, as the adult population becomes vaccinated against SARS-CoV-2, active infection and transmission will shift predominantly to the unvaccinated which includes children under age 12 who have not been eligible to receive vaccination at this point in time.

An unwell child living with a tracheostomy will most likely present to the ED if their caregiver has concerns about their condition. EDs are busy environments and, depending on the location of the hospital, may not have access to Otolaryngology cover full time. Staff can have varying levels of experience and expertise in paediatric care. Tertiary hospitals usually have an abundance of resources to help within specific specialty fields especially during normal working hours however, even in large academic institutions, care providers can be unfamiliar and lack confidence with regards to tracheostomy management, more so in the paediatric population ${ }^{15}$. This can result in hastily made decisions that, though well-meaning in their intent, may introduce a risk for iatrogenic injuries to the child and can unnecessarily increase aerosol generation to 
potentially expose healthcare workers.

The creation of our COVID-19 Emergency Management plan for paediatric tracheostomy patients was driven by the need for quality improvement when one of our patients had a larger tube inserted than appropriate for his airway in an attempt to reduce air leak and aerosol generation when they were coughing. We developed documents to provide a systematic approach and consistent advice about things to consider to deliver care safely for both the patient and the healthcare provider, irrespective of their location, resources or time of presentation. The objective was not only to provide support but to advance knowledge, improve communication and quality of care.

The personalised management plan for each patient was formulated with careful consideration of the individual's requirements, taking into account their specific anatomy, indication for tracheostomy, comorbidities, current tube dimensions and management. This was a consultative process between Paediatric Otolaryngology and the Clinical Nurse Consultant as tracheostomy tube nomenclature, branding, sizing, lengths and suitable conversion in the context of each patient's overall needs is highly specialised knowledge, especially in the infant population. Identifying how to troubleshoot around the existing tracheostomy tube to minimise air leak is emphasised as the first line approach. Recognising the situations for when to change the tube, if at all, was intended to reduce the risk of immediate and longer term damage to the stoma and airway from needless or panicked manipulation using an incorrect tube.

Tracheostomy tube change is not recommended as a routine whilst awaiting COVID-19 clearance due to potential high aerosol generation during the procedure however, should the need outweigh the risk, then understanding the safest way to achieve it with the correct tube readily available makes the process as streamlined and time efficient as possible. In well stocked tertiary hospitals that oversee complex paediatric patients, tracheostomy supplies may not be exhaustive or easy to locate from different departments after hours. Even if it is in stock, it may not be the correct conversion size. When patients integrate a prescribed alternative tube into their usual emergency kit, they facilitate efficient tube changing should the situation dictate and minimise the risk of an incorrect tube or size being placed. Our project is a relatively low cost initiative with multiple potential benefits for the child, their family and the health system in general.

Caregiver education about the plan and provision of a temporary alternative tracheostomy tube if appropriate, was devised to reduce anxiety and empower the carers should they need to present to hospital with concerns about COVID-19 or other respiratory tract illness during the pandemic. In many instances, these families are fearful about presenting to the ED, the risks of exposure for their child or themselves and potential need to change to a tube they had no experience with. The opportunity to explain and instruct them about these anticipated issues was extremely valuable. Familiarity about emergency management enables improved overall care and if required, a streamlined tube change reduces potential injury and the time with a leaking cuffed or uncuffed tube in-situ whilst the SARS-CoV-2 status is pending.

COVID-19 continues to have significant impact on healthcare throughout the world. It is possible new variants will evolve over time creating ongoing challenges for the general population and healthcare workers. It is therefore reasonable to have an emergency management plan such as ours for vulnerable patients with artificial airways to protect them and their care providers particularly in the situation of droplet and aerosol transmitted infections. Our initiative has been shared with other tertiary children's hospitals in Australia to improve the care for all paediatric patients living with tracheostomies during uncertain times. The longterm benefits of this COVID-19 emergency management plan extend beyond the current pandemic as the principles may be applied uniformly in the likely event of similar viral-based pandemics or outbreaks in the future.

\section{CONCLUSION}

A COVID-19 emergency management plan including a personalised recommendation for individual paediatric tracheostomy patients is presented for use in situations where SARS-CoV-2 infection status is unknown, suspected or confirmed. It has been designed to anticipate possible presentations, offer direction for troubleshooting and guide the steps in management if escalation in care is required. It provides a framework to 
reduce the risk of viral transmission to health care workers performing AGP on this cohort and decreases unnecessary manipulation and potential damage to the patient's airway. These simple and relatively inexpensive measures can improve the quality of care delivery with benefits to the patient, their families and the broader health system.

\section{Declaration of competing interest}

None.

\section{Acknowledgements}

The authors wish to acknowledge the contribution from the Children's Intensive Care Unit and Emergency Departments at Sydney Children's Hospital and Enable NSW.

\section{REFEERENCES}

1. Adil MT, Rahman R, Whitelaw D, Jain V, Al-Taan O, Rashid F, Munasinghe A, Jambulingam P. SARS-CoV-2 and the pandemic of COVID-19. Postgrad Med J. 2021 Feb;97(1144):110-116. doi: 10.1136/postgradmedj-2020-138386.

2. Wiersinga WJ, Rhodes A, Cheng AC, Peacock SJ, Prescott HC. Pathophysiology, Transmission, Diagnosis, and Treatment of Coronavirus Disease 2019 (COVID-19): A Review. JAMA. 2020 Aug 25;324(8):782-793. doi: 10.1001/jama.2020.12839.

3. Mick P, Murphy R. Aerosol-generating otolaryngology procedures and the need for enhanced PPE during the COVID-19 pandemic: a literature review. J Otolaryngol Head Neck Surg. 2020 May 11;49(1):29. doi: 10.1186/s40463-020-00424-7

4. Workman AD, Welling DB, Carter BS, Curry WT, Holbrook EH, Gray ST, Scangas GA, Bleier BS. Endonasal instrumentation and aerosolization risk in the era of COVID-19: simulation, literature review, and proposed mitigation strategies. Int Forum Allergy Rhinol. 2020 Jul;10(7):798-805. doi: 10.1002/alr.22577.

5. Lockhart SL, Duggan LV, Wax RS, Saad S, Grocott HP. Personal protective equipment (PPE) for both anesthesiologists and other airway managers: principles and practice during the COVID-19 pandemic. Can J Anaesth. 2020 Aug;67(8):1005-1015. doi: 10.1007/s12630-020-01673-w.

6. Thamboo A, Lea J, Sommer DD, Sowerby L, Abdalkhani A, Diamond C, Ham J, Heffernan A, Cai Long M, Phulka J, Wu YQ, Yeung P, Lammers M. Clinical evidence based review and recommendations of aerosol generating medical procedures in otolaryngology - head and neck surgery during the COVID-19 pandemic. J Otolaryngol Head Neck Surg. 2020 May 6;49(1):28. doi: 10.1186/s40463-020-00425-6.

7. Kana LA, Shuman AG, Helman J, Krawcke K, Brown DJ. Disparities and ethical considerations for children with tracheostomies during the COVID-19 pandemic. J Pediatr Rehabil Med. 2020;13(3):371376. doi: 10.3233/PRM-200749.

8. Praud JP. Long-Term Non-invasive Ventilation in Children: Current Use, Indications, and Contraindications. Front Pediatr. 2020 Nov 5;8:584334. doi: 10.3389/fped.2020.584334.

9. Sydney Children's Hospital Network. (2020, November 24). COVID-19 Standard Operating procedure, Emergency Tracheostomy - Non-Ventilated Patient. SCH. (No: S 94 v1)

10. Smith JD, Chen MM, Balakrishnan K, Sidell DR, di Stadio A, Schechtman SA, Brody RM, Kupfer RA, Rassekh CH, Brenner MJ. The Difficult Airway and Aerosol-Generating Procedures in COVID-19: Timeless Principles for Uncertain Times. Otolaryngol Head Neck Surg. 2020 Nov;163(5):934-937. doi: 10.1177/0194599820936615.

11. McGrath BA, Ashby N, Birchall M, Dean P, Doherty C, Ferguson K, Gimblett J, Grocott M, Jacob T, Kerawala C, Macnaughton P, Magennis P, Moonesinghe R, Twose P, Wallace S, Higgs A. Multidisciplinary guidance for safe tracheostomy care during the COVID-19 pandemic: the NHS National Patient Safety Improvement Programme (NatPatSIP). Anaesthesia. 2020 Dec;75(12):1659-1670. doi: 10.1111/anae.15120.

12. Pande RK, Bhalla A, Myatra SN, Yaddanpuddi LN, Gupta S, Sahoo TK, Prakash R, Sahu TA, Jain A, Gopal PB, Chaudhry D, Govil D, Dixit S, Samavedam S. Procedures in COVID-19 Patients: Part-I. 
Indian J Crit Care Med. 2020 Nov;24(Suppl 5):S263-S271. doi: 10.5005/jp-journals-10071-23597.

13. Gün E, Botan E, Özdemir H, Kendirli T. Successful treatment of COVID-19 infection in a child with tracheostomy. Tuberk Toraks. 2021 Jun;69(2):285-287. English. doi: 10.5578/tt.20219820.

14. Gray DM, Davies MA, Githinji L, Levin M, Mapani M, Nowalaza Z, Washaya N, Yassin A, Zampoli M, Zar HJ, Vanker A. COVID-19 and Pediatric Lung Disease: A South African Tertiary Center Experience. Front Pediatr. 2021 Jan 20;8:614076. doi: 10.3389/fped.2020.614076.

15. Ahmed ST, Yang C, Deng J, Bottalico DM, Matta-Arroyo E, Cassel-Choudhury G, Yang CJ. Implementation of an Online Multimedia Pediatric Tracheostomy Care Module for Healthcare Providers. Laryngoscope. 2021 Aug;131(8):1893-1901. doi: 10.1002/lary.29400.

\section{Hosted file}

Figure 1 - Tracheostomy management plan during COVID-19 pandemic.docx available at https: //authorea.com/users/445171/articles/544749-emergency-management-plan-for-paediatricpatients-with-tracheostomies-during-the-covid-19-pandemic

\section{Hosted file}

Figure 2 - Patient's personalised management plan during COVID-19 pandemic.docx available at https://authorea.com/users/445171/articles/544749-emergency-management-plan-forpaediatric-patients-with-tracheostomies-during-the-covid-19-pandemic

\section{Hosted file}

Figure 3 - Changing a tracheostomy tube for suspected or proven COVID-19 infection .docx available at https://authorea.com/users/445171/articles/544749-emergency-management-planfor-paediatric-patients-with-tracheostomies-during-the-covid-19-pandemic

\section{Hosted file}

Figure legends.docx available at https://authorea.com/users/445171/articles/544749-emergencymanagement-plan-for-paediatric-patients-with-tracheostomies-during-the-covid-19-pandemic 